\section{Catalysis-dependent stabilization of Bre1 fine-tunes histone H2B ubiquitylation to regulate gene transcription}

\author{
Glenn G. Wozniak ${ }^{1}$ and Brian D. Strahl ${ }^{1,2}$ \\ ${ }^{1}$ Curriculum in Genetics and Molecular Biology, ${ }^{2}$ Department \\ of Biochemistry and Biophysics, University of North Carolina \\ School of Medicine, Chapel Hill, North Carolina 27599, USA
}

Monoubiquitylation of histone H2B on Lys123 (H2BK123ub1) plays a multifaceted role in diverse DNA-templated processes, yet the mechanistic details by which this modification is regulated are not fully elucidated. Here we show in yeast that $\mathrm{H} 2 \mathrm{BK} 123 \mathrm{ub1}$ is regulated in part through the protein stability of the E3 ubiquitin ligase Bre1. We found that Bre1 stability is controlled by the Rtf1 subunit of the polymeraseassociated factor (PAF) complex and through the ability of Bre1 to catalyze H2BK123ub1. Using a domain in Rtf1 that stabilizes Bre1, we show that inappropriate Bre1 levels lead to defects in gene regulation. Collectively, these data uncover a novel quality control mechanism used by the cell to maintain proper Bre1 and H2BK123ub1 levels, thereby ensuring proper control of gene expression.

Supplemental material is available for this article.

Received April 7, 2014; revised version accepted July 8, 2014.

Histone post-translational modifications (PTMs) play essential roles in the regulation of chromatin structure and function (Kouzarides 2007; Zentner and Henikoff 2013). One such histone PTM that has been well studied as a regulator of multiple DNA-templated processes is monoubiquitylation of histone $\mathrm{H} 2 \mathrm{~B}$, which occurs at Lys123 (H2BK123ub1) in the budding yeast Saccharomyces cerevisiae (Robzyk et al. 2000). This PTM functions in the context of transcriptional regulation (both initiation and elongation) (Henry et al. 2003; Kao et al. 2004; Xiao et al. 2005; Pavri et al. 2006; Fleming et al. 2008; Chandrasekharan et al. 2009, 2010) but has also been linked to other processes, including DNA replication (Rizzardi et al. 2012; Trujillo and Osley 2012) and repair (Game and Chernikova 2009) and kinetochore function (Latham et al. 2011).

H2BK123ub1 functions in chromatin by several means. First, this mark physically alters chromatin compaction and nucleosome stability (Fleming et al. 2008; Chandrasekharan et al. 2009; Fierz et al. 2011). Another function of $\mathrm{H} 2 \mathrm{BK} 123 \mathrm{ub} 1$ is to promote histone $\mathrm{H} 3$ methylation at Lys4 (H3K4me) and Lys79 (H3K79me) in a mechanism of histone "cross-talk" referred to as trans-histone regulation (Briggs et al. 2002; Dover et al. 2002; Ng et al. 2002; Sun and Allis 2002). H3K4me

[Keywords: chromatin; gene transcription; histones; ubiquitylation] Corresponding author: brian_strahl@med.unc.edu

Article is online at http://www.genesdev.org/cgi/doi/10.1101/gad.243121.114. and $\mathrm{H} 3 \mathrm{~K} 79 \mathrm{me}$, in conjunction with $\mathrm{H} 2 \mathrm{BK} 123 \mathrm{ub} 1$, serve as markers of euchromatin and act to facilitate transcription factor recruitment and prevent the binding of silencing factors (Wozniak and Strahl 2014). Accordingly, loss of these PTMs leads to aberrant gene regulation.

In yeast, H2BK123ub1 is catalyzed by the concerted efforts of the ubiquitin-conjugating enzyme (E2) Rad6 and the RING finger domain-containing ubiquitin ligase (E3) Bre1 (Robzyk et al. 2000; Hwang et al. 2003; Wood et al. 2003a). Similar to other E3 ligases, Brel serves as the substrate recognition module for the complex and is important for the recruitment of Rad6 to chromatin (Wood et al. 2003a). Studies have also found that the polymeraseassociated factor (PAF) complex associates with Rad6 and facilitates its recruitment to gene bodies ( $\mathrm{Ng}$ et al. 2003; Wood et al. 2003b; Xiao et al. 2005). Although the mechanistic underpinnings of this recruitment are not entirely clear, it is known that the Rtf1 subunit of the PAF complex plays a major role (Wood et al. 2003b; Xiao et al. 2005). In addition to Bre1 recruitment and catalysis, H2BK123ub1 levels are also controlled by the deubiquitylases Ubp8 and Ubp10 (Henry et al. 2003; Emre et al. 2005). Loss of Ubp8 or Ubp10 leads to phenotypes similar to the loss of H2BK123ub1, indicating that the levels of this PTM are carefully regulated in the cell.

In this study, we found that $\mathrm{H} 2 \mathrm{BK} 123 \mathrm{ub} 1$ is regulated through the control of Brel protein stability. Surprisingly, Bre1 stability is primarily controlled through its catalytic activity in addition to its association with the PAF complex that is likely responsible for its recruitment to chromatin. By taking advantage of a region in Rtfl of the PAF complex that can stabilize Bre1, we found that inappropriate stabilization of Brel under normal conditions leads to defects in gene regulation. Our results suggest a "rheostat" control mechanism for H2BK123ub1 that contributes to proper transcriptional control.

\section{Results and Discussion}

Trans-histone regulatory pathways facilitate H2BK123ub1

A major mechanism by which histone modifications are regulated is via trans-histone pathways, which involve a histone region or histone PTM regulating the outcome of another histone modification in an intranucleosomal or internucleosomal manner. For example, methylation of $\mathrm{H} 3$ at Lys 36 (H3K36me) is regulated by regions within $\mathrm{H} 2 \mathrm{~A}$ and $\mathrm{H} 4$, which form a nucleosomal surface that the responsible enzyme, Set2, binds to when catalyzing this mark (Du and Briggs 2010). H2BK123ub1, on the other hand, regulates the outcome of $\mathrm{H} 3 \mathrm{~K} 4$ and $\mathrm{H} 3 \mathrm{~K} 79$ methylation in a trans-histone pathway that controls the function of the Set1 and Dot1 enzymes, respectively. However, trans-histone pathways controlling the outcome of H2BK123ub1 have not been fully explored.

(C) 2014 Wozniak and Strahl This article is distributed exclusively by Cold Spring Harbor Laboratory Press for the first six months after the full-issue publication date (see http://genesdev.cshlp.org/site/misc/terms. xhtml). After six months, it is available under a Creative Commons License (Attribution-NonCommercial 4.0 International), as described at http:// creativecommons.org/licenses/by-nc/4.0/. 
To identify regulatory mechanisms important for the outcome of H2K123ub1, we determined the levels of H2BK123ub1 as well as the downstream modification H3K79me3 in yeast strains lacking the N-terminal tails of histones $\mathrm{H} 3, \mathrm{H} 4, \mathrm{H} 2 \mathrm{~A}$, and $\mathrm{H} 2 \mathrm{~B}$. As expected, loss of the $\mathrm{H} 4$ tail eliminated $\mathrm{H} 3 \mathrm{~K} 79 \mathrm{me} 3$ without affecting H2BK123ub1 (Fig. 1A; Fingerman et al. 2007). We also observed two opposing effects on H2BK123ub1. First, loss of the $\mathrm{H} 2 \mathrm{~B}$ tail reduced the levels of $\mathrm{H} 2 \mathrm{~B}$ without affecting $\mathrm{H} 2 \mathrm{BK} 123 \mathrm{ub1}$, suggesting that a higher proportion of $\mathrm{H} 2 \mathrm{~B}$ in these cells is ubiquitylated (Fig. 1A). Second, and consistent with a previous report (Zheng et al. 2010), we found that loss of the $\mathrm{H} 2 \mathrm{~A}$ tail reduced $\mathrm{H} 2 \mathrm{BK} 123 \mathrm{ub} 1$ as well as H3K79me3 (Fig. 1A). Residues 16-20 of H2A (the HAR domain) primarily mediate this regulation, since deletion of this domain affects H2BK123ub1 and H3K79me3 to an extent similar to that of loss of the entire tail (Fig. 1B; Supplemental Fig. 1). Taken together, our data verify that multiple trans-histone pathways exist to regulate H2BK123ub1.

\section{The HAR domain regulates H2BK123ub1 by stabilizing Bre1}

We next sought to uncover the mechanism by which the HAR domain regulates H2BK123ub1. Given the close physical proximity of the HAR domain to H2BK123 on the nucleosomal surface (Fig. 1C), we hypothesized that the HAR domain may play a role in the ubiquitylation reaction itself. Thus, we investigated whether loss of the HAR domain had any effect on the E2 or E3 ubiquitin ligases Rad6 and Bre1, respectively. Loss of the HAR

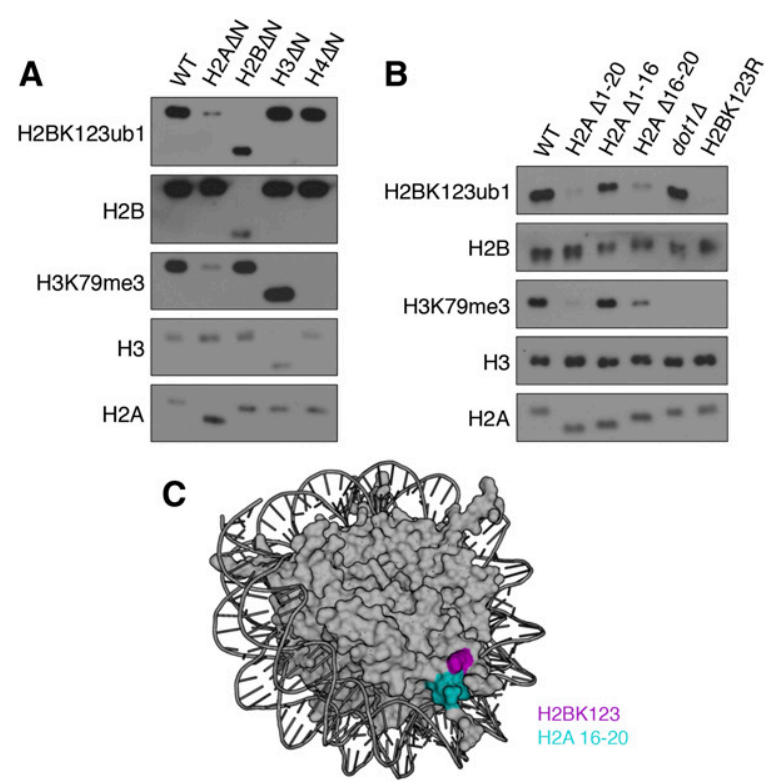

Figure 1. The H2A N-terminal tail regulates H2BK123 ubiquitylation. (A) Shown is a Western screen of histone methylation and ubiquitylation states in wild-type (WT) and mutant strains lacking the N-terminal tails of each of the core histones. (H2A $\Delta \mathrm{N}) \Delta 1-20$; $(\mathrm{H} 2 \mathrm{~B} \Delta \mathrm{N}) \Delta 1-32 ;(\mathrm{H} 3 \Delta \mathrm{N}) \Delta 1-30 ;(\mathrm{H} 4 \Delta \mathrm{N}) \Delta 1-27$. (B) Residues $16-20$ of histone $\mathrm{H} 2 \mathrm{~A}$ (HAR domain) regulate H2BK123ub1 and H3K79me3. Strains harboring the indicated truncations of $\mathrm{H} 2 \mathrm{~A}$ were probed for ubiquitylation and methylation states as in $A$. (C) The HAR domain (cyan) is located next to H2BK123 (magenta) on the surface of the nucleosome (Protein Data Bank [PDB] ID: 1ID3). domain did not alter either total or bulk chromatin-bound levels of Rad6 (Supplemental Fig. 2). To assess Bre1 levels, we transformed bre $1 \Delta$ strains either containing or lacking the HAR domain with a low-copy plasmid expressing ADH1-driven, N-terminally Flag-tagged Bre1. Importantly, this expression construct restores H2BK123ub1 to wild-type levels in the bre1 $\Delta$ strain and behaves similarly to a version containing the native BRE1 promoter (Fig. 2A; Supplemental Fig. 3). Surprisingly, the levels of Bre1 in the HAR deletion strain were reduced, matching the decrease in H2BK123ub1 (Fig. 2A). Moreover, this was not the result of decreased $B R E 1$ transcription as measured by RT-PCR (Supplemental Fig. 4), indicating that the HAR domain regulates Bre1 levels through a mechanism that is post-transcriptional.

\section{Bre1 stability is dependent on its ability to ubiquitylate H2BK123}

Given the possibility that the HAR domain might regulate Bre1 stability through its contribution to a nucleosomal surface required by Bre 1 to catalyze H2BK123ub1, we next asked whether the loss of H2BK123ub1 itself might also regulate Bre1 stability. Strikingly, we found that Bre1 protein levels were nearly abolished in strains harboring a point mutation at $\mathrm{H} 2 \mathrm{BK} 123$ (H2BK123R) (Fig. 2B). As with the loss of the HAR domain, the H2BK123R mutation did not affect BRE1 expression, suggesting that the regulation occurs at the level of the protein stability (Supplemental Fig. 4). Consistent with this, a cyclohexamide $(\mathrm{CHX})$ pulse-chase analysis revealed that Bre1 is more rapidly turned over in the $\mathrm{H} 2 \mathrm{BK} 123 \mathrm{R}$ strain (Fig. 2C, cf. wild-type and H2BK123R at 30 min after CHX treatment). Taken together, these data provide strong support that Bre1 in the HAR $\Delta$ and H2BK123R strains is subject to post-transcriptional control. We note that Bre1 regulation does not appear to involve the proteasome, since MG132 treatment failed to stabilize Bre1 (Supplemental Fig. 5). This result is in agreement with another report showing that MG132 decreases H2BK123ub1 levels (Mimnaugh et al. 1997).

We next ascertained whether mutations in the ubiquitylation machinery would also affect Brel stability. We found that loss of Rad6, like the H2BK123R mutant, also decreased Bre1 levels (Fig. 2B). Moreover, both deletion of the catalytic RING finger domain of Bre1 (1-650) and a point mutation that disrupts its enzymatic function (H665A) (Wood et al. 2003a) destabilize Bre1 (Fig. 2D). Additionally, RING finger mutants of Bre1 also had a destabilizing effect on the protein when expressed in the context of wild-type endogenous Bre1, indicating that destabilization is not merely the consequence of a global loss of histone ubiquitylation (Fig. 2D). Thus, the ability of Bre1 to ubiquitylate chromatin is important for its stability.

\section{The PAF complex contributes to Bre1 stability via a conserved domain in Rtf1}

Given that Brel stability is dependent on catalysis, we next sought to determine whether other proteins that promote H2BK123ub1 also regulate Bre1 stability. We focused on the PAF complex, which has been well studied as a regulator of H2BK123ub1 (Jaehning 2010). As shown in Figure $3 \mathrm{~A}$, deletions of individual members of the complex have varying effects on H2BK123ub1, with the 
A

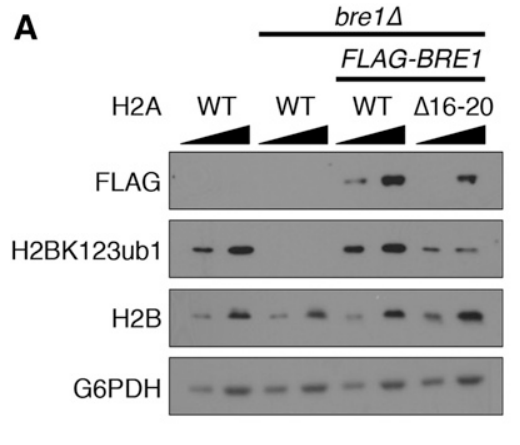

B

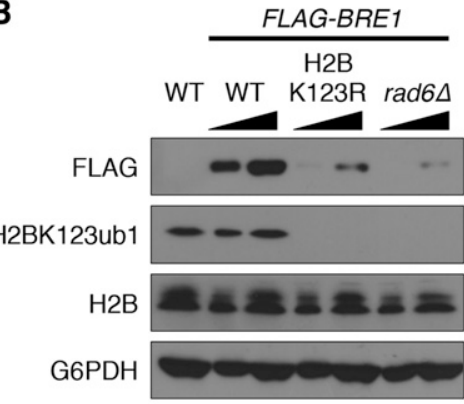

C

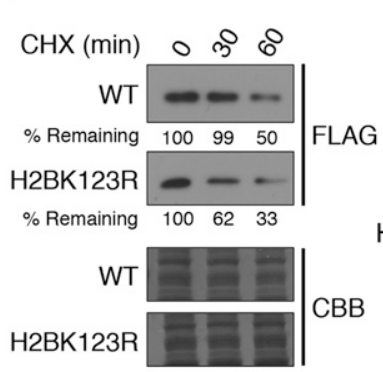

D

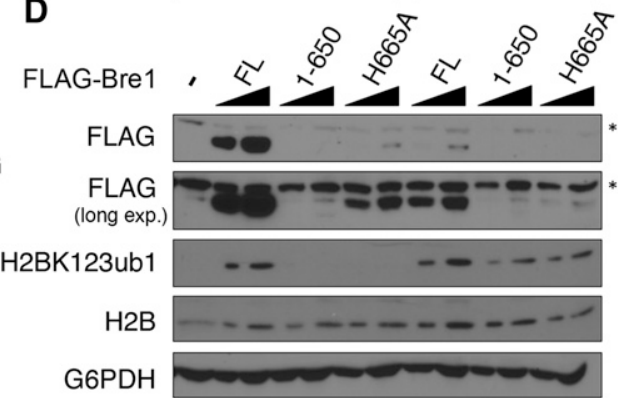

Figure 2. Brel stability is dependent on the catalysis of H2BK123ub1. (A) The HAR domain is important for the stability of Brel. The indicated mutant strains were transformed with empty vector or ADH1-driven Flag-BRE1 and subjected to immunoblot analysis with the indicated antibodies. G6PDH served as a loading control. Increasing amounts of extract were loaded for each sample, as indicated by solid black triangles. $(B)$ Catalysis of H2BK123ubl is required for Brel stability. The indicated strains were analyzed as in $A$. (C) Loss of H2BK123ub1 destabilizes Bre1. Wild-type (WT) and H2BK123R strains were treated with cyclohexamide (CHX) for the indicated amount of time. Samples taken at each time point were analyzed by immunoblot analysis and Coomassie Brilliant Blue (CBB) staining. The percentage of signal compared with the 0 -min time point for each sample is indicated (\% remaining). (D) The RING finger domain of Bre1 is required for stability. Wild-type or bre1 $\Delta$ strains expressing empty vector $(-)$, full-length (FL) Flag-Bre1, or mutant derivatives lacking the RING finger domain (1-650) or harboring an inactivating point mutation (H665A) were analyzed by immunoblot analysis. $\left(^{\star}\right)$ Nonspecific band.

paf1s and rtf1s strains having the strongest effect. Significantly, we found that the loss of H2BK123ub1 correlates with the loss of Bre1 levels in these mutant strains, thereby linking the PAF complex to Bre1 stability and H2BK123ub1.

Rtf1 is the only subunit of the PAF complex that is absolutely required for H2BK123ub1 ( Ng et al. 2003; Wood et al. 2003b; Xiao et al. 2005). This appears to be mediated by a small conserved domain of Rtf1 called the histone modification domain (HMD), which is capable of facilitating H2BK123ub1 independently of the PAF complex (Piro et al. 2012). Based on this finding, we hypothesized that the HMD promotes H2BK123ub1 by stabilizing Bre1. To test this idea, we coexpressed Myctagged HMD fused to a nuclear localization sequence (NLS-Myc-HMD) and Flag-Brel in the rtf1 strain. In agreement with published data (Piro et al. 2012), we found that the HMD could restore H2BK123ub1 in the rtf1D strain (Fig. 3B). Moreover, we found that expression of the HMD could also rescue Brel levels, indicating a critical role for the HMD in stabilizing Bre1.

To examine the functional relevance of HMD-mediated Brel stabilization, we investigated its role in telomeric silencing, a function linked to both Brel and Rtf1. We made use of a telomeric silencing reporter strain that has the URA3 gene inserted near the telomere of chromosome VII. Loss of Rtf1 in this strain shows a severe growth defect when grown on medium containing 5-FOA, indicating a loss of silencing (Fig. 3C). In line with the finding that the HMD could rescue Brel levels and H2BK123ub1, expression of the HMD was able to restore the silencing defect of the $r t f 1 \Delta$ strain (Fig. 3C). These data demonstrate that the HMD plays an important role in gene silencing by stabilizing Bre1.

\section{Altering the balance of Bre1 leads to defects in gene regulation}

The ability of the HMD to stabilize Brel allowed us to use it as a tool to ask why Brel is under such careful regulation. To address this question, we again used the telomeric silencing reporter strain used above. In this strain, we overexpressed Brel from the highly expressed GPD promoter either alone or in combination with the HMD and measured growth on 5-FOA. Overexpression of Bre1 alone did not result in any growth defect on 5-FOA (Fig. 4A), consistent with inability of Brel overexpression to increase the levels of H2BK123ub1 (Fig. 2D). In contrast, we found that overexpression of the HMD resulted in reduced growth on 5-FOA, and this effect was exacerbated when Brel was also overexpressed, indicating loss of silencing of the URA3 reporter (Fig. 4A). In validation of the reporter strain, we also observed increased transcription of two naturally silenced subtelomeric genes (YFR057W [chromosome VI] and COS12 [chromosome VII]) with Brel stabilization, indicating that aberrant levels of Bre1 impact transcription of normally silenced telomere-proximal genes (Fig. 4B).

Last, we sought to determine whether the observed changes in gene expression were the result of HMD-mediated binding of Bre1 at telomeres. To determine this, we performed chromatin immunoprecipitation (ChIP) to measure Brel binding to a subtelomeric region of chromosome VI proximal to YFR057W, where the HMD has been previously shown to bind (Piro et al. 2012). In agreement with the up-regulation of YFR057W, we found increased Brel binding in this region in the presence of the HMD (Fig. 4C). Taken together, these observations demonstrate that aberrant stabilization of Bre1 at telomeres leads to defects in gene silencing. Given that loss of Ubp8 and Ubp10 also results in increased H2BK123ub1 levels at euchromatic and telomeric regions (Henry et al. 2003; Emre et al. 2005), the collective data support a model in which the ubiquitylation machinery is present across the genome but is kept in check by the opposing functions of RNA polymerase II (RNAPII)-dependent PAF recruitment and the deubiquitylating enzymes that reduce H2BK123ub1, both of which would control Bre1 stability and hence H2BK123ub1 levels genome-wide.

\section{Concluding remarks}

In this study, we uncover a novel pathway of H2BK123ub1 regulation that involves the precise control of Bre1 protein 


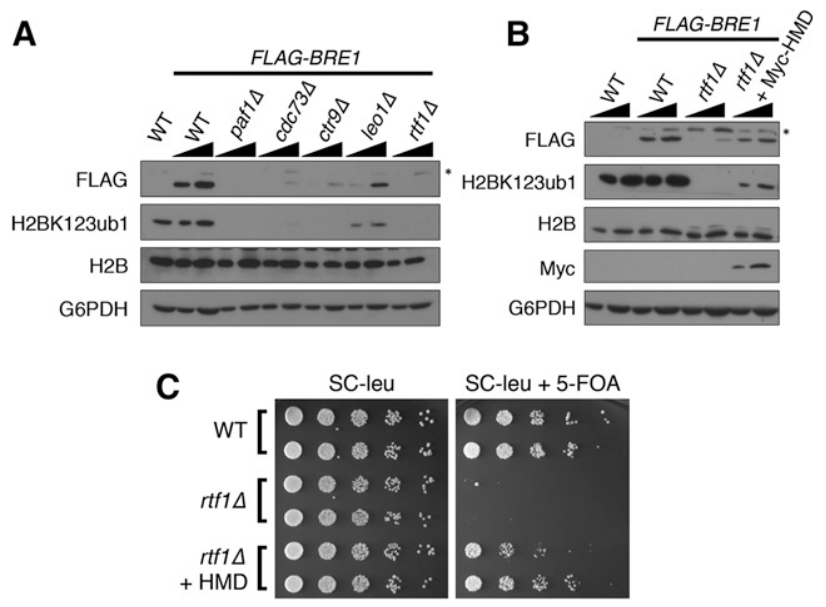

Figure 3. The histone modification domain (HMD) of Rtfl stabilizes Bre1. $(A)$. The PAF complex regulates Brel stability. The indicated strains, transformed with empty vector or Flag-BRE1, were subjected to immunoblot analysis. Increasing amounts of extract were loaded for each sample, as indicated by solid black triangles. $\left(^{*}\right)$ Nonspecific band. $(B)$ The HMD of Rtfl stabilizes Brel. The indicated strains were transformed with Flag-BRE1 and/ or NLS-Myc-HMD (Myc-HMD, CEN) and subjected to immunoblot analysis as in $A$. $(C)$ The HMD is sufficient for mediating the telomeric silencing function of Rtf1. Empty vector or a plasmid expressing NLS-Myc-HMD (HMD, 2 $\mu$ ) were transformed into wildtype (WT) or $r t f 1 \Delta$ telomeric silencing reporter strains harboring the $U R A 3$ gene inserted within a subtelomeric region of chromosome VII. Strains were plated on SC-leu medium with or without 5-FOA.

stability. Using mutants that disrupt (1) the nucleosomal surface targeted by Bre1, (2) Bre1 catalytic activity, or (3) proteins that aid in Brel catalysis (i.e., Rad6 and the PAF complex), we show that the ability to ubiquitylate H2B is critical for the stabilization of this E3 ligase. By expressing a domain in Rtf1 that couples the PAF complex with Brel and leads to its stabilization, we show that aberrant Bre1 levels result in adverse consequences for gene silencing. Taken together, these findings reveal a novel control mechanism for Brel that we suggest functions to fine-tune the appropriate levels of H2BK123ub1 genomewide.

In addition to the regulation of Bre1, another mechanism that acts to fine-tune the levels of H2BK123ub1 across the genome is the deubiquitylating enzymes Ubp8 and Ubp10. A question remains as to why the cell would use two distinct mechanisms to control H2BK123ub1 levels. Perhaps, similar to histone acetylases and deacetylases, where the equilibrium of the "on" and "off" enzymes define the precise levels of histone acetylation at any given point across the genome, it may be that the level of H2BK123ub1 across the genome is similarly governed by the equilibrium of Rad6/Brel and Ubp8/ Ubp10. Consistent with this idea, deletion of the heterochromatin-associated Ubp10 deubiquitylase results in increased levels of H2BK123ub1 in silenced regions of the genome (Emre et al. 2005). This finding implies that Bre1/Rad6 can localize to these regions but is prevented from functioning by the removal of H2BK123ub1. Notably, we were unable to detect Brel at a subtelomeric region of chromosome VI under normal conditions (Fig. 4C), suggesting that it may interact transiently with these regions. In contrast, within transcribed regions where
Bre1 is stabilized by the PAF complex, the equilibrium shifts toward productive H2BK123ub1 (Fig. 5A). Thus, a possible surveillance mechanism comprising the deubquitylating enzymes ensures loss of Bre1 and erasure of H2BK123ub1 where it would otherwise drive inappropriate functions (Fig. 5B).

Our observations also provide insight into the regulation of H2BK123ub1 by the PAF complex. Previous work has shown that Brel directly interacts with the PAF complex in vitro using purified recombinant proteins (Kim and Roeder 2009). In addition, we demonstrated that Rad6/Brel is associated with the PAF complex in yeast (Xiao et al. 2005). Given these observations, we propose that the PAF complex, through the HMD, stabilizes Brel in transcribed regions, which in turn promotes Rad6 recruitment (Wood et al. 2003a) and H2BK123ub1 (Fig. 5A). It is not entirely clear how a potential interaction with Rtfl could stabilize Bre1, but the interaction may either mask specific degradation sequences within Brel or aid in the recruitment of Brel to its nucleosomal substrate, which may be the actual stabilizing interaction.

One of the important mechanistic functions of H2BK123ub1, in addition to promoting nucleosomal disruption and stability during transcription elongation,
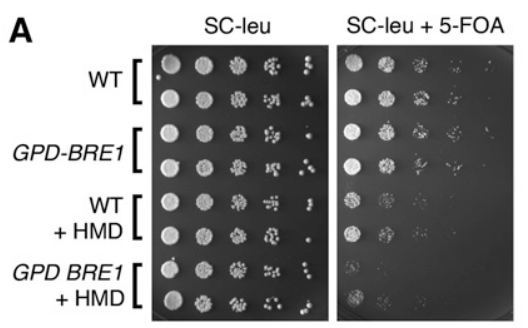

B

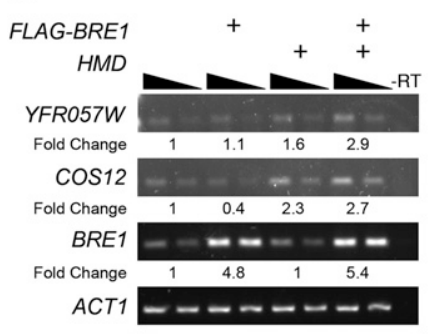

C

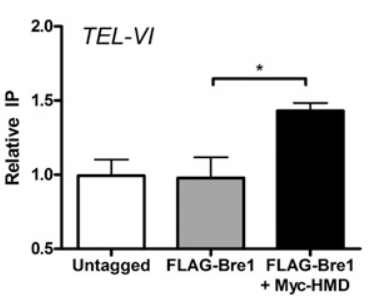

Figure 4. Aberrant Brel stabilization disrupts gene silencing. (A) Stabilization of Brel causes defective silencing at telomeres. Telomeric silencing strains that overexpressed Brel from the GPD promoter $(G P D-B R E)$ and/or the HMD $(2 \mu)$ were used and analyzed as in Figure 3C. $(B)$ Stabilized Brel alters the expression of naturally silenced telomeric genes. RT-PCR was performed with RNA isolated from strains expressing Flag-BRE1 (CEN) and/or the HMD $(2 \mu)$ with primers directed toward the subtelomeric genes YFR057W (chromosome VI), COS12 (chromosome VII), and BRE1 or the housekeeping gene ACT1. Decreasing amounts of cDNA were used for each PCR, as indicated by solid black triangles. The expression of each target was normalized to ACT1, and the fold change versus wild-type (WT) was calculated and is shown below each strain. (C) The HMD recruits Brel to telomeres. ChIP was performed with M2 Flag agarose under each of the indicated conditions. ChIP and input DNA were used as template for PCR reactions containing primers specific to a subtelomeric region of chromosome VI (TEL$V I)$. Relative immunoprecipitation represents fold change enrichment versus untagged. See the Supplemental Material for further details. Data represent mean $\pm \operatorname{SEM}(n=3) .\left(^{*}\right) P<0.04$. 
A

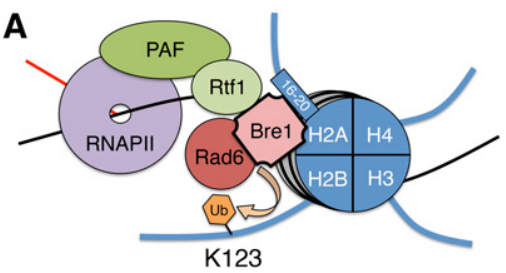

B

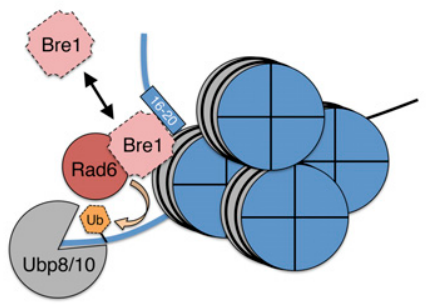

Figure 5. Transcription-coupled stabilization of Brel fine-tunes H2B ubiquitylation. We found that the Rtf1 subunit of the PAF complex is important for stabilizing Brel and promoting H2BK123ub1. Given the close association of the PAF complex with transcribing RNAPII, we propose that Rtf1, residues 16-20 of histone H2A, and perhaps other proteins associated with the transcriptional apparatus interact with and stabilize Brel (indicated by solid black outline) to promote H2BK123ubl in active regions of the genome. Once transcription is complete or in repressed regions, the absence of the transcriptional machinery leads to Brel instability (indicated by dashed outline). Transient interactions of Brel/Rad6 with chromatin in repressed regions catalyze short-lived H2BK123ub1 (dashed outline) that is rapidly removed by the deubiquitylating enzymes Ubp8/10.

is the regulation of histone methylation at $\mathrm{H} 3 \mathrm{~K} 4$ and H3K79 (Briggs et al. 2002; Dover et al. 2002; Ng et al. 2002; Sun and Allis 2002; Chandrasekharan et al. 2010). This form of histone "cross-talk" has been the focus of numerous studies over the past decade, but the mechanism remains to be fully elucidated. Two primary models exist, which suggest that $\mathrm{H} 2 \mathrm{BK} 123 \mathrm{ub} 1$ acts as either a wedge in chromatin to facilitate enzyme access (Fierz et al. 2011) or a bridge to the histone methyltransferases (either directly [McGinty et al. 2008; Kim et al. 2013] or indirectly [Lee et al. 2007; Vitaliano-Prunier et al. 2008]). The indirect recruitment mechanism has been proposed to involve Cps35/Swd2, which is a subunit of the H3K4-methylating COMPASS complex and has been suggested to interact with the H3K79 methyltransferase Dot1 (Lee et al. 2007). However, both of these models share the common theme that the ubiquitin moiety itself at H2BK123 mediates the "cross-talk." Intriguingly, our data demonstrate that the same mutations used to characterize H2BK123ub1-mediated "cross-talk" also disrupt the stability of Bre1. Thus, it will be intriguing to determine whether any aspect of the trans-histone pathway of H3K4 and H3K79 methylation might involve Brel itself independent of H2BK123ub1. In support of this idea, Bre1 has been shown to interact with Cps35/Swd2 in vivo (VitalianoPrunier et al. 2008), and, intriguingly, mutations that disrupt H2BK123ub1 (and hence Brel stability) also disrupt the ability of Cps35/Swd2 to facilitate COMPASSmediated H3K4 methylation (Lee et al. 2007; VitalianoPrunier et al. 2008). Thus, Cps35/Swd2 may be a link between Brel and H3K4 methylation. Future studies will be required to revisit some of the basic assumptions of H2BK123ub1-mediated histone "cross-talk" and the details that underlie Brel regulation.

\section{Materials and methods}

Yeast strains and plasmids

Strains and plasmids used in this study are listed in Supplemental Tables 1 and 2. Gene disruptions and endogenous overexpression were performed as previously described (Janke et al. 2004) and verified by both PCR and immunoblotting.

\section{Yeast whole-cell extracts and Western blot analysis}

Yeast were grown in YPD or synthetic complete dropout (SC) medium at $30^{\circ} \mathrm{C}$ to mid-log phase, and extracts were prepared as previously described (Mehta et al. 2010). Antibodies and dilutions are listed in the Supplemental Material.

\section{Phenotypic spotting assays}

Fivefold serial dilutions of saturated overnight yeast cultures were plated on YPD or SC medium with or without the indicated drugs. Cells were plated at a starting $\mathrm{OD}_{600}$ of 0.5 on the appropriate medium and imaged after 2-4 d of growth at $30^{\circ} \mathrm{C}$.

\section{RNA isolation and RT-PCR}

RNA was prepared from $10 \mathrm{OD}_{600}$ units of mid-log phase cells using hot acid phenol-chloroform extraction followed by ethanol precipitation. Crude RNA was DNaseI-treated (Promega) and then purified using an RNeasy minikit (Qiagen). cDNA was synthesized using SuperScript II first strand synthesis system (Life Technologies) and diluted 1/10 prior to amplification by PCR. Primers are listed in Supplemental Table 3. Reactions were run on $2 \%$ agarose gels and visualized by UV with SYBR Safe DNA gel stain (Life Technologies). Bands were quantified using ImageJ software.

\section{ChIP}

ChIP was performed as described previously (Jha and Strahl 2014) with some exceptions. Sonication for each sample was performed for $20 \mathrm{~min}$ with alternating on/off cycles of $30 \mathrm{sec}$ using a Bioruptor Standard (Diagenode). Immunoprecipitation was performed overnight with $1 \mathrm{mg}$ of clarified, sonicated extract and $20 \mu \mathrm{L}$ of equilibrated Flag M2 agarose (Sigma). Primer sequences are listed in Supplemental Table 3, and analysis methodologies are described further in the Supplemental Material.

\section{Acknowledgments}

We thank members of the Strahl laboratory for a critical reading of the manuscript. We also thank David Allis, Elizabeth Gjoneska, Judith Jaehning, Michael Parra, Ali Shilatifard, Jeff Smith, Zu-Wen Sun, and Fred Winston for yeast strains and plasmids. This work was supported by a National Science Foundation grant to B.D.S. (grant no. 1330320).

\section{References}

Briggs SD, Xiao T, Sun ZW, Caldwell JA, Shabanowitz J, Hunt DF, Allis CD, Strahl BD. 2002. Gene silencing: trans-histone regulatory pathway in chromatin. Nature 418: 498.

Chandrasekharan MB, Huang F, Sun ZW. 2009. Ubiquitination of histone $\mathrm{H} 2 \mathrm{~B}$ regulates chromatin dynamics by enhancing nucleosome stability. Proc Nat1 Acad Sci 106: 16686-16691.

Chandrasekharan MB, Huang F, Sun ZW. 2010. Histone H2B ubiquitination and beyond: regulation of nucleosome stability, chromatin dynamics and the trans-histone $\mathrm{H} 3$ methylation. Epigenetics 5: 460-468.

Dover J, Schneider J, Tawiah-Boateng MA, Wood A, Dean K, Johnston M, Shilatifard A. 2002. Methylation of histone H3 by COMPASS requires ubiquitination of histone H2B by Rad6. J Biol Chem 277: 28368-28371.

Du HN, Briggs SD. 2010. A nucleosome surface formed by histone H4, $\mathrm{H} 2 \mathrm{~A}$, and $\mathrm{H} 3$ residues is needed for proper histone $\mathrm{H} 3$ Lys36 
methylation, histone acetylation, and repression of cryptic transcription. J Biol Chem 285: 11704-11713.

Emre NC, Ingvarsdottir K, Wyce A, Wood A, Krogan NJ, Henry KW, Li K, Marmorstein R, Greenblatt JF, Shilatifard A, et al. 2005. Maintenance of low histone ubiquitylation by Ubp10 correlates with telomereproximal Sir2 association and gene silencing. Mol Cell 17: 585-594.

Fierz B, Chatterjee C, McGinty RK, Bar-Dagan M, Raleigh DP, Muir TW. 2011. Histone H2B ubiquitylation disrupts local and higher-order chromatin compaction. Nat Chem Biol 7: 113-119.

Fingerman IM, Li HC, Briggs SD. 2007. A charge-based interaction between histone $\mathrm{H} 4$ and Dot1 is required for H3K79 methylation and telomere silencing: identification of a new trans-histone pathway. Genes Dev 21: 2018-2029.

Fleming AB, Kao CF, Hillyer C, Pikaart M, Osley MA. 2008. H2B ubiquitylation plays a role in nucleosome dynamics during transcription elongation. Mol Cell 31: 57-66.

Game JC, Chernikova SB. 2009. The role of RAD6 in recombinational repair, checkpoints and meiosis via histone modification. DNA Repair 8: 470-482.

Henry KW, Wyce A, Lo WS, Duggan LJ, Emre NC, Kao CF, Pillus L, Shilatifard A, Osley MA, Berger SL. 2003. Transcriptional activation via sequential histone $\mathrm{H} 2 \mathrm{~B}$ ubiquitylation and deubiquitylation, mediated by SAGA-associated Ubp8. Genes Dev 17: 2648-2663.

Hwang WW, Venkatasubrahmanyam S, Ianculescu AG, Tong A, Boone C, Madhani HD. 2003. A conserved RING finger protein required for histone H2B monoubiquitination and cell size control. Mol Cell 11: 261-266.

Jaehning JA. 2010. The Paf1 complex: platform or player in RNA polymerase II transcription? Biochim Biophys Acta 1799: 379-388.

Janke C, Magiera MM, Rathfelder N, Taxis C, Reber S, Maekawa H, Moreno-Borchart A, Doenges G, Schwob E, Schiebel E, et al. 2004. A versatile toolbox for PCR-based tagging of yeast genes: new fluorescent proteins, more markers and promoter substitution cassettes. Yeast 21: 947-962.

Jha DK, Strahl BD. 2014. An RNA polymerase II-coupled function for histone H3K36 methylation in checkpoint activation and DSB repair. Nat Commun 5: 3965.

Kao CF, Hillyer C, Tsukuda T, Henry K, Berger S, Osley MA. 2004. Rad6 plays a role in transcriptional activation through ubiquitylation of histone H2B. Genes Dev 18: 184-195.

Kim J, Roeder RG. 2009. Direct Brel-Paf1 complex interactions and RING finger-independent Brel-Rad6 interactions mediate histone H2B ubiquitylation in yeast. J Biol Chem 284: 20582-20592.

Kim J, Kim JA, McGinty RK, Nguyen UT, Muir TW, Allis CD, Roeder RG. 2013. The n-SET domain of Setl regulates H2B ubiquitylationdependent H3K4 methylation. Mol Cell 49: 1121-1133.

Kouzarides T. 2007. Chromatin modifications and their function. Cell 128: $693-705$.

Latham JA, Chosed RJ, Wang S, Dent SY. 2011. Chromatin signaling to kinetochores: transregulation of Dam1 methylation by histone H2B ubiquitination. Cell 146: 709-719.

Lee JS, Shukla A, Schneider J, Swanson SK, Washburn MP, Florens L, Bhaumik SR, Shilatifard A. 2007. Histone crosstalk between H2B monoubiquitination and $\mathrm{H} 3$ methylation mediated by COMPASS. Cell 131: 1084-1096.

McGinty RK, Kim J, Chatterjee C, Roeder RG, Muir TW. 2008. Chemically ubiquitylated histone $\mathrm{H} 2 \mathrm{~B}$ stimulates hDot1L-mediated intranucleosomal methylation. Nature 453: 812-816.

Mehta M, Braberg H, Wang S, Lozsa A, Shales M, Solache A, Krogan NJ, Keogh MC. 2010. Individual lysine acetylations on the $\mathrm{N}$ terminus of Saccharomyces cerevisiae H2A.Z are highly but not differentially regulated. J Biol Chem 285: 39855-39865.

Mimnaugh EG, Chen HY, Davie JR, Celis JE, Neckers L. 1997. Rapid deubiquitination of nucleosomal histones in human tumor cells caused by proteasome inhibitors and stress response inducers: effects on replication, transcription, translation, and the cellular stress response. Biochemistry 36: 14418-14429.

Ng HH, Xu RM, Zhang Y, Struhl K. 2002. Ubiquitination of histone H2B by Rad6 is required for efficient Dot1-mediated methylation of histone H3 lysine 79. J Biol Chem 277: 34655-34657.

Ng HH, Dole S, Struhl K. 2003. The Rtfl component of the Paf1 transcriptional elongation complex is required for ubiquitination of histone H2B. J Biol Chem 278: 33625-33628.
Pavri R, Zhu B, Li G, Trojer P, Mandal S, Shilatifard A, Reinberg D. 2006. Histone H2B monoubiquitination functions cooperatively with FACT to regulate elongation by RNA polymerase II. Cell 125: 703-717.

Piro AS, Mayekar MK, Warner MH, Davis CP, Arndt KM. 2012. Small region of Rtf1 protein can substitute for complete Pafl complex in facilitating global histone H2B ubiquitylation in yeast. Proc Natl Acad Sci 109: 10837-10842.

Rizzardi LF, Dorn ES, Strahl BD, Cook JG. 2012. DNA replication origin function is promoted by H3K4 di-methylation in Saccharomyces cerevisiae. Genetics 192: 371-384.

Robzyk K, Recht J, Osley MA. 2000. Rad6-dependent ubiquitination of histone H2B in yeast. Science 287: 501-504.

Sun ZW, Allis CD. 2002. Ubiquitination of histone H2B regulates H3 methylation and gene silencing in yeast. Nature 418: 104-108.

Trujillo KM, Osley MA. 2012. A role for H2B ubiquitylation in DNA replication. Mol Cell 48: 734-746.

Vitaliano-Prunier A, Menant A, Hobeika M, Geli V, Gwizdek C, Dargemont C. 2008. Ubiquitylation of the COMPASS component Swd2 links H2B ubiquitylation to H3K4 trimethylation. Nat Cell Biol 10: 1365-1371.

Wood A, Krogan NJ, Dover J, Schneider J, Heidt J, Boateng MA, Dean K, Golshani A, Zhang Y, Greenblatt JF, et al. 2003a. Bre1, an E3 ubiquitin ligase required for recruitment and substrate selection of Rad6 at a promoter. Mol Cell 11: 267-274.

Wood A, Schneider J, Dover J, Johnston M, Shilatifard A. 2003b. The Paf1 complex is essential for histone monoubiquitination by the Rad6Brel complex, which signals for histone methylation by COMPASS and Dotlp. J Biol Chem 278: 34739-34742.

Wozniak GG, Strahl BD. 2014. Hitting the 'mark': interpreting lysine methylation in the context of active transcription. Biochim Biophys Acta doi: 10.1016/j.bbagrm.2014.03.002.

Xiao T, Kao CF, Krogan NJ, Sun ZW, Greenblatt JF, Osley MA, Strahl BD. 2005. Histone $\mathrm{H} 2 \mathrm{~B}$ ubiquitylation is associated with elongating RNA polymerase II. Mol Cell Biol 25: 637-651.

Zentner GE, Henikoff S. 2013. Regulation of nucleosome dynamics by histone modifications. Nat Struct Mol Biol 20: 259-266.

Zheng S, Wyrick J, Reese JC. 2010. Novel trans-tail regulation of H2B ubiquitylation and $\mathrm{H} 3 \mathrm{~K} 4$ methylation by the $\mathrm{N}$ terminus of histone H2A. Mol Cell Biol 30: 3635-3645. 


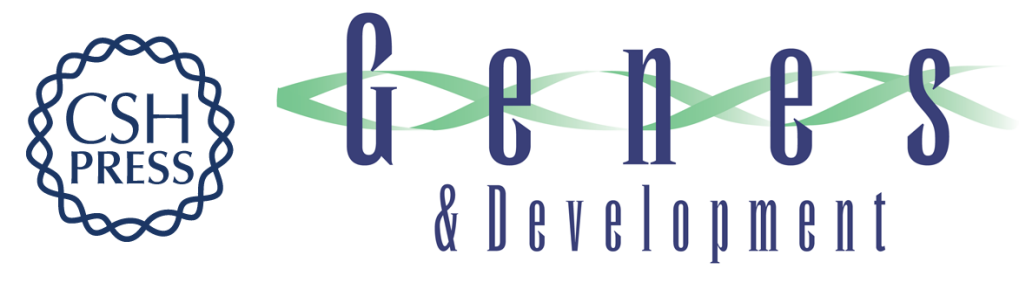

\title{
Catalysis-dependent stabilization of Bre1 fine-tunes histone H2B ubiquitylation to regulate gene transcription
}

\author{
Glenn G. Wozniak and Brian D. Strahl
}

Genes Dev. 2014, 28:

Access the most recent version at doi:10.1101/gad.243121.114

\section{Supplemental http://genesdev.cshlp.org/content/suppl/2014/07/31/28.15.1647.DC1 Material}

References This article cites 39 articles, 16 of which can be accessed free at: http://genesdev.cshlp.org/content/28/15/1647.full.html\#ref-list-1

Creative This article is distributed exclusively by Cold Spring Harbor Laboratory Press for the first Commons six months after the full-issue publication date (see

License http://genesdev.cshlp.org/site/misc/terms.xhtml). After six months, it is available under a Creative Commons License (Attribution-NonCommercial 4.0 International), as described at http://creativecommons.org/licenses/by-nc/4.0/.

Email Alerting Receive free email alerts when new articles cite this article - sign up in the box at the top Service right corner of the article or click here.

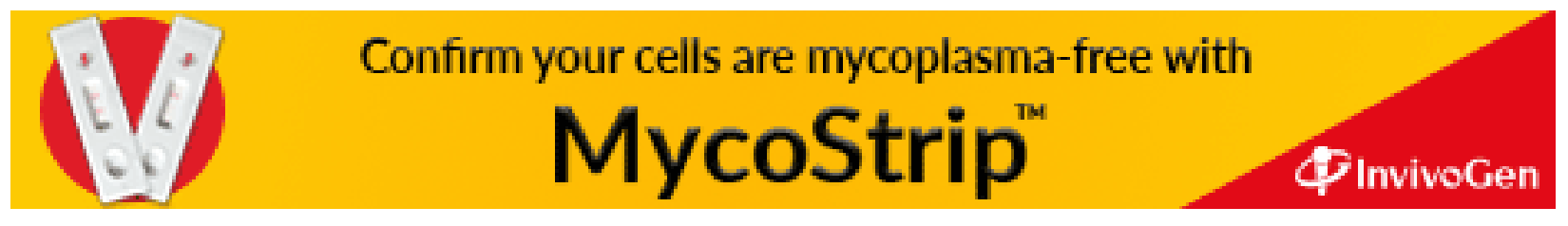

